\title{
A YOUNG PERSON'S VIEW OF THE SUPERCONDUCTING SUPER COLLIDER
}

\author{
Andy Moya* \\ Superconducting Super Collider Laboratory ${ }^{\dagger}$ \\ 2550 Beckleymeade Ave. \\ Dallas, TX 75237
}

August 1990

\section{DISCLAIMER}

\begin{abstract}
This report was prepared as an account of work sponsored by an agency of the United States Government. Neithei the United States Government nor any agency thereof, nor any of their employees, makes any warranty, express or implied, or assumes any legal liability or responsibility for the accuracy, completeness, or usefulness of any information, apparatus, product, or process disclosed, or represents that its use would not infringe privately owned rights. Reference herein to any specific commercial product, process, or service by trade name trademark, manufacturer, or otherwise does not necessarily constitute or imply its endorsement, recommendation, or favoring by the United States Government or any agency thereof. The views and opinions of authors expressed herein do not necessarily state or reflect those of the United States Government or any agency thereof.
\end{abstract}

* Student Intern for the Conventional Construction Division, Summer 1990.

†Operated by the Universities Research Association, Inc., for the U.S. Department of Energy under Contract No. DE-AC02-89ER40486. 


\section{What is the SSC?}

In today's world, modern science plays a major role in society. Various scientific discoveries have made an impact on the lives of many people. In order to encounter such discoveries, experiments must be conducted. These experiments sometimes require a number of sophisticated and costly scientific instruments. This is one reason why the Superconducting Super Collider (SSC) is required. This highly complicated instrument will play a vital part in the discovery of new fundamental particles of matter unknown to man at this moment.

The SSC will be the world's largest scientific instrument. Its working mechanism will be a high-energy proton-proton accelerator and collider, consisting of two rings of counter-rotating proton beams of very high intensity, housed in an underground tunnel 54 miles long, which will collide in several given areas. Each beam of protons contains an energy of $20 \mathrm{TeV}$ (Trillion Electron Volts); during the collisions a total energy of $40 \mathrm{TeV}$ is created. By observing what occurs during the collisions, scientists hope to study the fundamental particles of matter, and the physical laws that govern them by observing what occurs during the collisions. These observations will be made in the various underground detector halls known as the interaction regions. These are the heart of what will be the world's largest and most technologically advanced instrument: the SSC.

\section{Why is the SSC Necessary?}

In order to answer this question we must begin by breaking the field of science down. Science consists of many areas, most of which are dependent up $n$ work in the other areas of science. Medicine and other components of thie medical field, as well as biology, are based on the experiments conducied in the area of chemistry and its branches. In turn, chemistry is based upon physics, and physics is centered around the study of the fundamental particles of matter. However, in order to study these tiny, vital particles of matter, a series of high energy tests and experiments must be conducted. This is the reason why the SSC is necessary. With the use of such a large instrument, the physicists will be able to extend results already achieved at a larger scale. 
When the atom was first hypothesized, it was believed to be the fundamental particle of all matter. However, actual experimentation provided information leading to the concept that atoms could be broken down into smaller substances. Scientists soon discovered that atoms consist of a nucleus and small components called electrons which revolve around the nucleus. The nucleus was found to be the center of all of the atom's activity. This satisfied the curiosity of most people, but it was not long before the quest to discover more of the unknown was aroused again. More experiments were conducted, and it was discovered that the nucleus is composed of more minute particles, protons, positively charged, and neutrons, without charge (electrons and protons have opposite charges). The scientists and the public again were satisfied, but there were still a number of people who wanted to continue the quest. How much more complicated could this microscopic atom be? The search continued with a vast number of highly complicated experiments conducted. An interesting new discovery was made. The proton was found to be made up of various even smaller particles known as quarks. There are several different types of quarks. They are paralleled by another group of constituents known as leptons, of which the electron is a member. The quarks and their associated leptons are believed to come in "families" as follows:

- "up" quark, "down" quark, electron, and electron neutrino;

- "charmed" quark, "strange" quark, muon, and muon neutrino;

- "top" quark, "buttorn" quark, tau lepton, and tau neutrino.

Presently, only five types of quarks are krown to exist. The sixth quark. the top quark (one of whose parallels is the tau neutrino), is believed to exist, but not confirmed. Detecting it is one of the goals of the SSC. With this great utensil, scientists hope to discover this component and observe its properties.

For the listed reasons, and various others not mentioned, we can see why the SSC is necessary. After all, who knows what could exist beyond the quark? 


\section{How is the SSC Operated?}

The SSC is a superconducting proton-proton accelerator and collider. It consists of two rings, each containing special magnets for guiding proton beams which will collide at several points. Unfortunately, it is not as easy as it sounds. One must go through a number of steps before reaching the point at which one is ready for the collisions.

The protons which the SSC will use come from the hydrogen atom. An electron is added to the normally neutral atom, and then this negatively charged "ion" is accelerated to a velocity that is so high, that all of the electrons can be stripped off, and only the positively charged protons are left. These protons then pass through several smaller accelerators before they aciually enter the collider ring itself.

The first accelerator is called the LINAC, which stands for Linear Accelerator. It is a straight accelerator which will propel the protons to an energy of 600 million electron volts (MeV). They are then shot into a circular accelerator, called the LEB ( Low Energy Booster). Here, the protons are repeatedly sent in a circular path, speeding up each time around, until an energy of 12 billion electron volts, usually expressed as giga electron volts $(\mathrm{GeV})$, is reached. As soon as this energy ${ }_{i} \mathrm{i}$ s achieved, they enier the MEB (Medium Energy Booster). In the MEB, they are propelled in a similar manner until they reach a total energy of $200 \mathrm{GeV}$. After this, they enter the last of the boosters, which is called the HEB (High Energy Booster). Here thev are accelerated until they reach an energy of $2 \mathrm{TeV}$. The protons are now released into the collider main ring.

The main ring of the collider is the largest collider ring in the world. It is approximately 54 miles in length, housed in a circular tunnel, approximately 200 feet below ground in Ellis County, Texas. As the protons enter the ring, they possess an energy of $2 \mathrm{TeV}$, and the magnetic field in the superconducting magnets that will guide them is 0.66 Tesla. They are now accelerated until they reach $20 \mathrm{TeV}$, during which time the guiding magnetic field rises from 0.6 lesla to 6.6 Tesla. Once this energy has been achieved, the beams are aimed at each other, and the collisions occur. These collisions 
occur at a rate of over 100 million collisions per second. It is impossible for humans to perceive the resulting events, so electronic detectors placed in interaction halls record the results instead. A total of four interaction region detector halls are in the present plans of the SSC, and more can be added in the future. These are the vital components of the SSC, and are vital to the sophisticated process by which the supercollider works.

\section{Where and How is it Constructed?}

The Superconducting Super Collider is a very detailed and delicate project. This is the reason why a perfect site had to be chosen as the home for this $\$ 8$ Billion project. When it was first announced that there were plans to construct such a project, many states were interested in becoming the host for this endeavor, and submitted proposals to the Department of Energy (DOE), who will fund the supercollider project. Then, the selection process began. After much consideration, the selection was narrowed down to eight finalists, one of which was the state of Texas. The department then conducted many geotechnical experiments and studies of the finalists, in order to determine which site would be the best for this structure. In 1988, a decision was finally reached, and the site was announced. The location for the Superconducting Super Collider would be near Waxahachie in Ellis County, Texas, as shown in Figure 1.

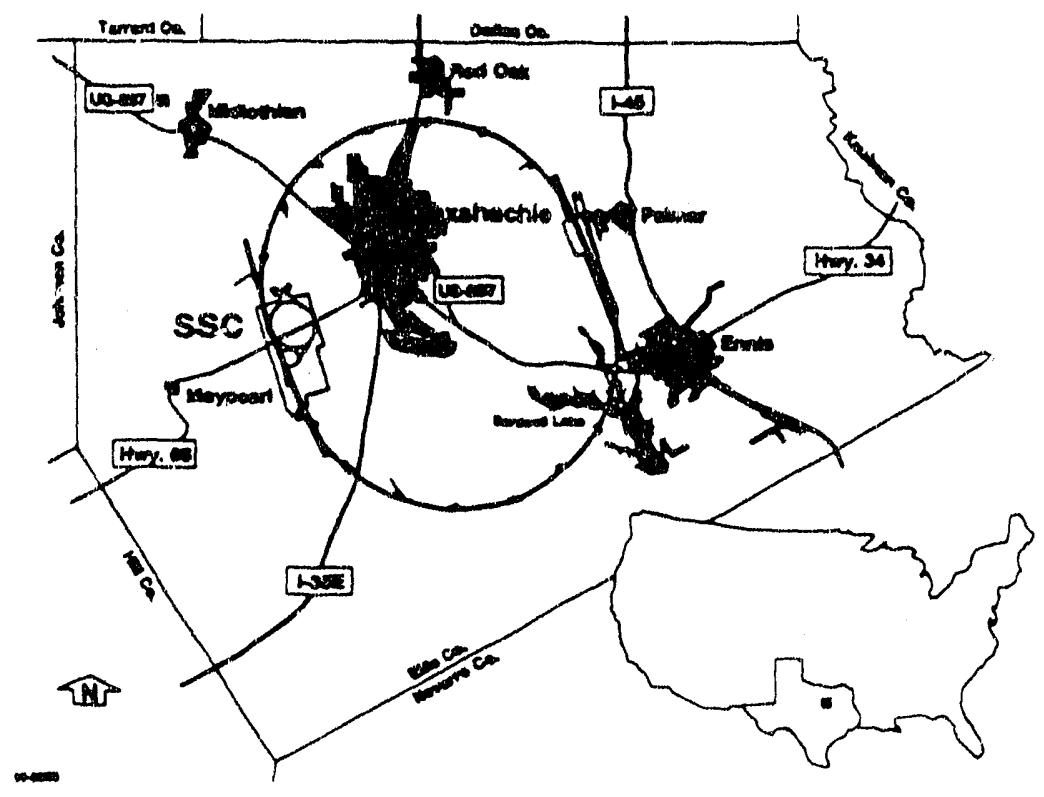

Figure 1. SSC Location. 


\section{Location}

Waxahachie is a town located approximately thirty minutes south of the Dallas-Fort Worth area. With a population of approximately twenty thousand, it is a small, yet moderately modern city. One of the major attractions of Waxahachie is its historic homes. These homes, built during the Victorian time period (1800's), are featured annually during the first weekend in June in what is known as "The Gingerbread Trail", which is a tour of the historic houses registered by the state of Texas. These houses also attract the movie industry to Waxahachie as well. At one time, the city was nicknamed "Little Hollywood", because of the number of movies which were filmed during the 80's, such as "Places In The Heart", "Tender Mercies", "Return To Peyton Place", and many others. Despite having this interesting bit of trivia, the town and its environs also has a geology which is most desirable for a project like the SSC. Below the surface of Ellis County is a substance known as Austin Chalk. The rest of the area not containing the Austin chalk contains either Taylor Marl or Eagle Ford Shale. Most of these materials are excellent elements for boring and constructing underground. This is the main reason why Ellis County was chosen as the home of the Superconducting Super Collider.

\section{Accelerators and the Main Ring}

Before the protons are ready to enter the main ring of the collider, they pass through a linear accelerator and three smaller circular accelerators. It is within these accelerators that the velocity of the moving particles is increased, step by step. The LINAC and two of the smaller circular accelerators are housed in tunnels constructed by what is known as "cut-and-cover"

operations. They will not be placed as deep in the ground as the main ring will be.

\section{The LINAC}

The linear accelerator (LINAC) is the smallest of the SSC accelerators. Just before it is a so-called ion source in which hydrogen atoms are changed to a state of being ionized. The hydrogen ions enter the LINAC and are accelerated from a speed corresponding to an energy of $2.5 \mathrm{MeV}$ to $600 \mathrm{MeV}$. 
As this energy is reached, the ions are stripped of their electrons, and only the protons remain. They now continue to the next accelerator.

\section{The LEB}

The protons enter the Low Energy Booster (LEB), the first of the circular accelerators with an energy of $600 \mathrm{MeV}$. By the time they are ready to move on to the next booster, an energy of $12 \mathrm{GeV}$ is achieved.

\section{The MEB}

Traveling at an energy of $12 \mathrm{GeV}$, the protons encer what is called the Medium Energy Booster, or the MEB. This is the second circular booster. Again, the protons are accelerated to a higher energy. Within the MEB, the acceleration increases the energy to $200 \mathrm{GeV}$. The protons now move to the last of the minor accelerators.

\section{The HEB}

The third and last of the circular accelerators, the High Energy Booster (HEB), augments the energy of the protons until they reach $2 \mathrm{TeV}$. As soon as this energy is reached, the protons will move directly into the main ring, in which the collisions will occur.

\section{The Collider Ring}

The protons are now in the final stage before the collision. As they enter the ring with an energy of $2 \mathrm{TeV}$, they are repeatedly sent in a circular path until they finally achieve the energy of $20 \mathrm{TeV}$. It is now that the beams are aimed at each other, and the collisions begin to take place.

\section{Technical Components}

In order to operate, the SSC must have many technical components. The two main components requiring modern technology are the magnets for guiding the protons and the detectors which are required in order to conduct the experiments. 


\section{Magnets}

The magnets used by the SSC are superconducting magnets. They use special concuctors which, when cooled to liquid helium temperatures $(-452$ degrees F), loose every trace of electrical resistance. These magnets still require electricity in order to produce the magnetic field which will be used during the operation. (The magnets on your refrigerator are not superconducting.) The SSC will use two types of magnets: quadrupoles and dipoles.

\section{Dipole Magnets}

The dipnle magnets will be used as bending mignets in the SSC. They contain only two poles, as the name denotes, and they will be used as bending magnets. They will guide the protons within the vacuum chamber or bend the beams around their 54-mile circular paths.

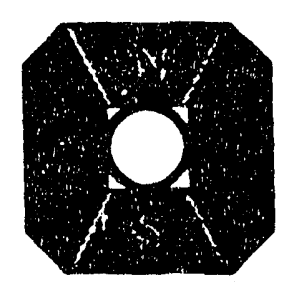

Figure 2. Dipole Magnet.

\section{Quadrupole Magnets}

The quadrupole magnets, magnets with four poles, will be used as the focusing magnets at the SSC. As the beam of protons passes through the vacuum chamber, the magnetic fields interact to focus the beam at the very center of the chamber. This keeps the beam from colliding with the walls of the vacuum chamber. 


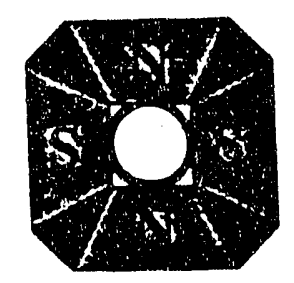

Figure 3. Quadrupole Magnet.

\section{Detectors}

As mentioned earlier, the collisions in the main collider ring will be occurring at a rate of over 100 million collisions per second. It is virtually impossible for humans to sense these collisions, so technology now steps in. The SSC will use electronic detectors to record the results of each interaction. These detectors are very complicated and sophisticated machines, and will be the largest in the world. In the following picture, we can see the comparison of how small an average-sized man is compared to the immense detectors used in the SSC.

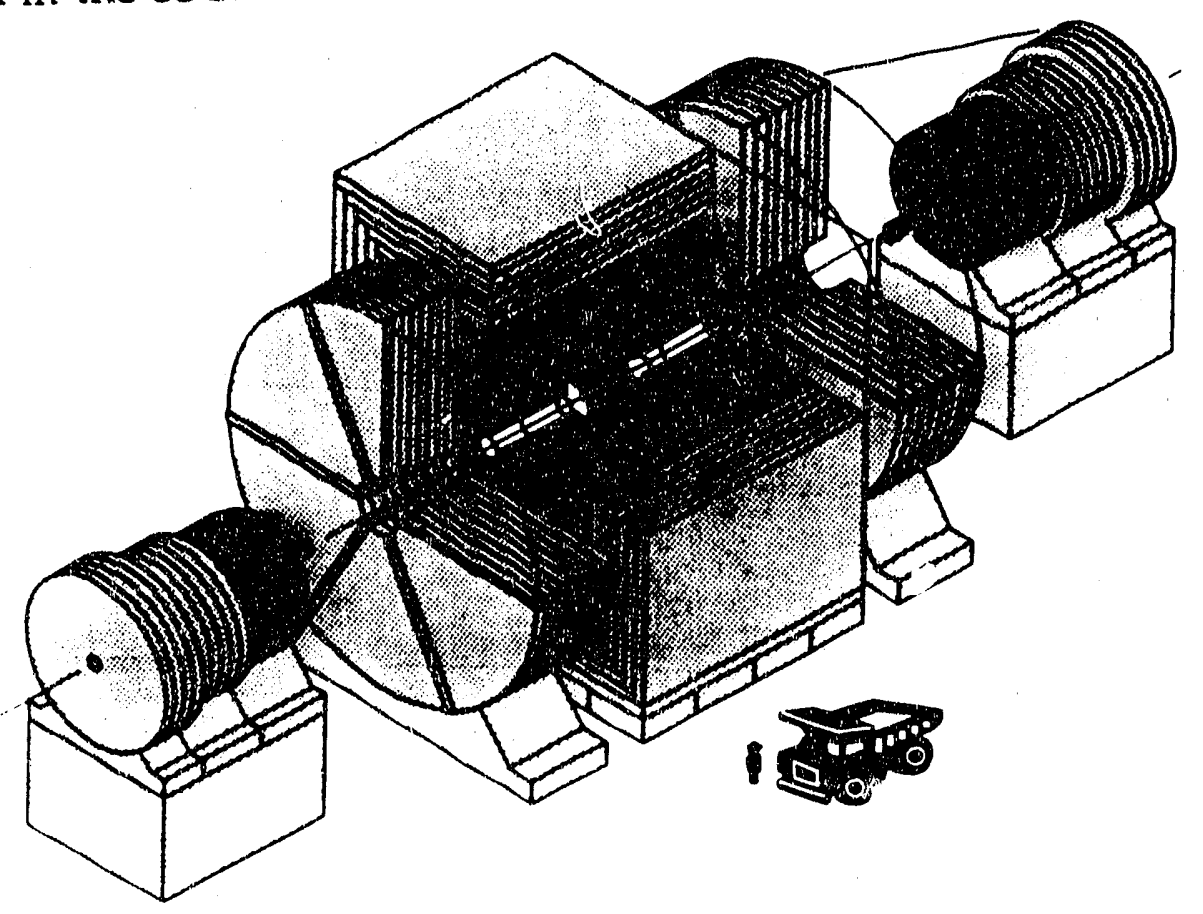

Figure 4. One type of detector.

These detectors will be located in what are called detector halls. These will be huge structures placed far beneath the surface of the Earth. 


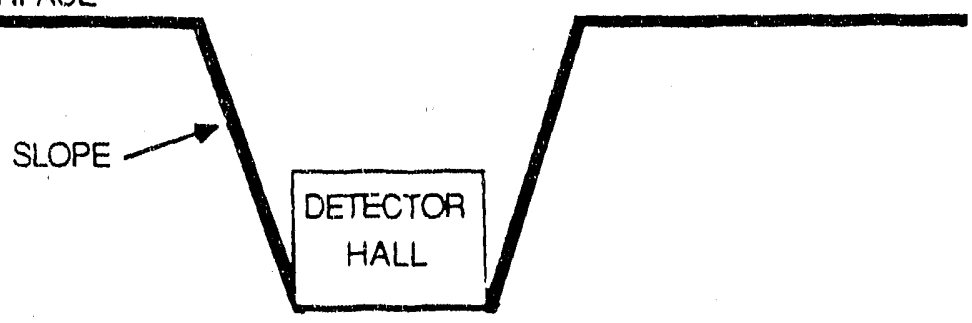

Figure 5. Detector hall.

The SSC will be the world's largest scientific project. Thus, it will require quite a bit of work. The other two collider projects, the Tevatron in Illinois (a proton-antiproton collider) and the LEP (an electron-positron collider in Europe), are not as large as this grand project will be. That is why the total construction cost of this project is $\$ 8$ billion. It will be the world's largest scientific instrument.

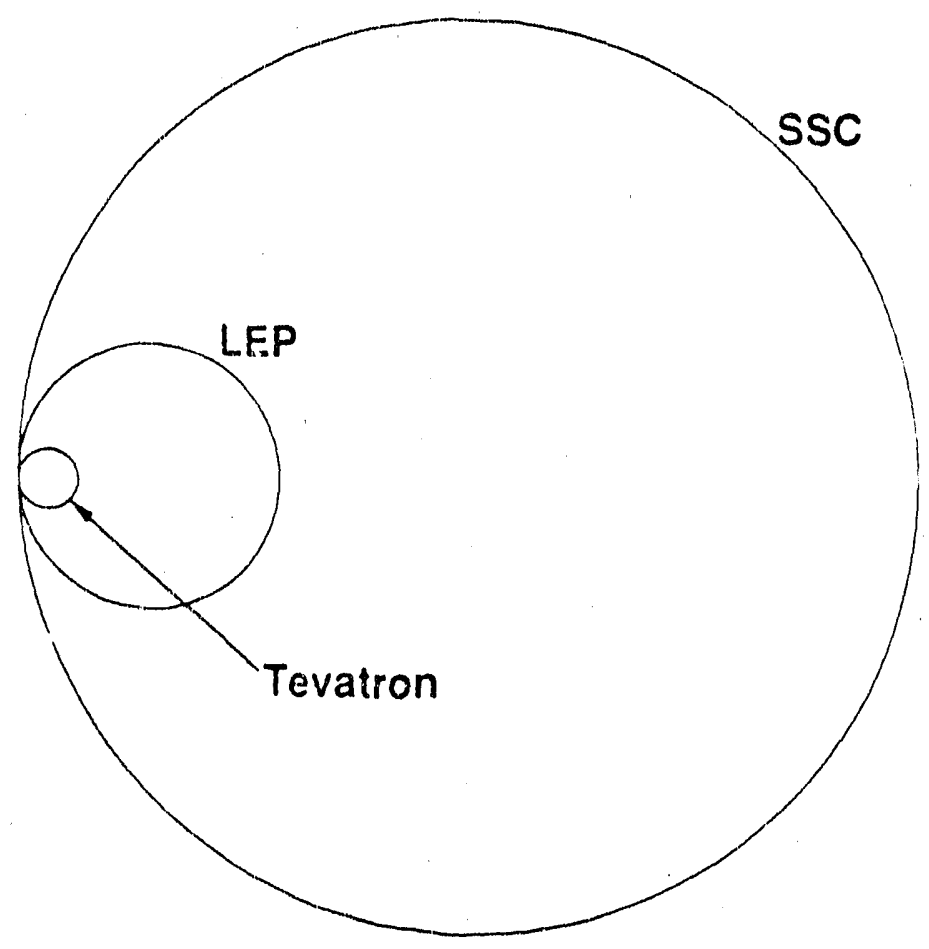

Figure 6. Collider comparison. 


\section{What Are the Effects on Society?}

With a project the size of the SSC, people often wonder what the effects upon society will be. Such is the case with this endeavor.

The Superconducting Super Collider is not a military project, as sometimes imagined. For instance, the SSC cannot be used for producing chemical warfare weapons or nuclear arms, an idea of concern to some people. Thus it will not present a military danger to the surrounding area.

Another concern to many people is the concept of radiation being emitted from the collisions. Obviously, some radiation will be produced, but only when the accelerator is operating, and it will be dissipated in the shielding and rock formations covering the tunnel. But that cannot be prevented. Any residual radiation appearing at surface level will not present a danger to the citizens or crops in the surrounding area. Such radiation is a small fraction of the natural background radiation. It is routinely produced in existing high energy physics laboratories, and is routinely monitored and carefully controlled to ensure the effects involved comply with existing federal regulations and guidelines. The results of ongoing tests confirm that the amount reieased is so minute, it is less than the radiation released by many other common everyday objects, including natural radioactive substances in the human body. This situation is not unprecedented. At Fermi National Laboratory (Fermilab), located in the outskirts of Chicago, Illinois, the Tevatron, a proton-antiproton collider, releases a form of radiation similar to that which will be released by the SSC. The Tevatron has been in operation for over five years, and the geology and topography around the Tevatron is very similar to that surrounding the SSC. In fact, corn crops are actually grown right above the Tevatron itself. In the entire time that the Tevatron has been operational, there have been no reports of the emitted radiation causing damage to any of the surrounding life, or any reported damage to any of the surrounding crops. Thus, as can be seen, the SSC will not constitute a radiation hazard to the public or environment.

The SSC will not create an image which will affect the beauty of the surrounding area. It will be an underground structure, and the only objects visible from an aerial view, in addition to the buildings of the central 
laboratory campus, will be the service stations located periodically along the path of the tunnel. There are also plans to use several cooling ponds on the campus and in some service areas. These, in fact, will impart a beauty to the area, as well as provide an environment for wildlife such as fish and water birds. Therefore, the SSC project will be doing more to help the area than it will be doing harm.

There are also other benefits that the Superconducting Super Collider will provide. For example, various educational programs will be made available which will undoubtedly be of benefit to local schools. There are also programs already started, which give students who have an interest in science the chance to experience real science firsthand. During the summer of 1990, the first student intern program was established. A total of 29 students were selected from a group of 200 applicants. These students spent eight weeks in an internship, learning from scientists, what the various aspects of working in a science environment are like. A plan to continue these summer opportunities is under discussion, along with plans to possibly form a co-op program for graduate students. At the moment, personnel from the SSC are setting up workshops for teachers in the surrounding area. These workshops will give teachers an insight on what the Super Collider will do. It is anticipated that the instructors will encourage students of every level to take an interest in science. These are just a few of the many educational programs which the SSC will provide.

If the Superconducting Super Collider is successful, it will make the United States a world leader in the field of science. Along with ensuring new fundamental discoveries, it will create a number of jobs for people in the surrounding area. At the present time (summer of 1990), a total of over 600 people are employed by the SSC. (This figure includes physicists, engineers, and supporting personnel.) Once the collider is complete, that number will more than double. This will be both a cultural and economic benefit to the people in the areas around the SSC.

Thus, as can be concluded, the Superconducting Super Collider will bring various effects upon society, many of which will be beneficial to the citizens in the surrounding area, and it will be a major step in a forefront area of science. 


\section{Acicnowledgernents}

Most of the information in this publication was taken from various sources. Some of the material was taken from lectures given to the 1990 SSC interns. The breakdown of the field of science, locatsd in the section titled "Why Is The SSC Necessary?," was taken frnm the introduction of To the Heart of Matter . The Superconducting Super Collider (released by the Universities Research Association in Washington D.C.). Thanks should also be expressed to Dr. Larry Coulson and Dr. Sam Baker of the SSC Laboratory for their comments and consideration. I would also like to express a special thanks to Dale Montana for her taking the time to edit the publication. A very big word of thanks is also given to my mentor, Per Dahl, for his guidance and care. His editing and comments were very much appreciated and valued in the writing of this paper. 

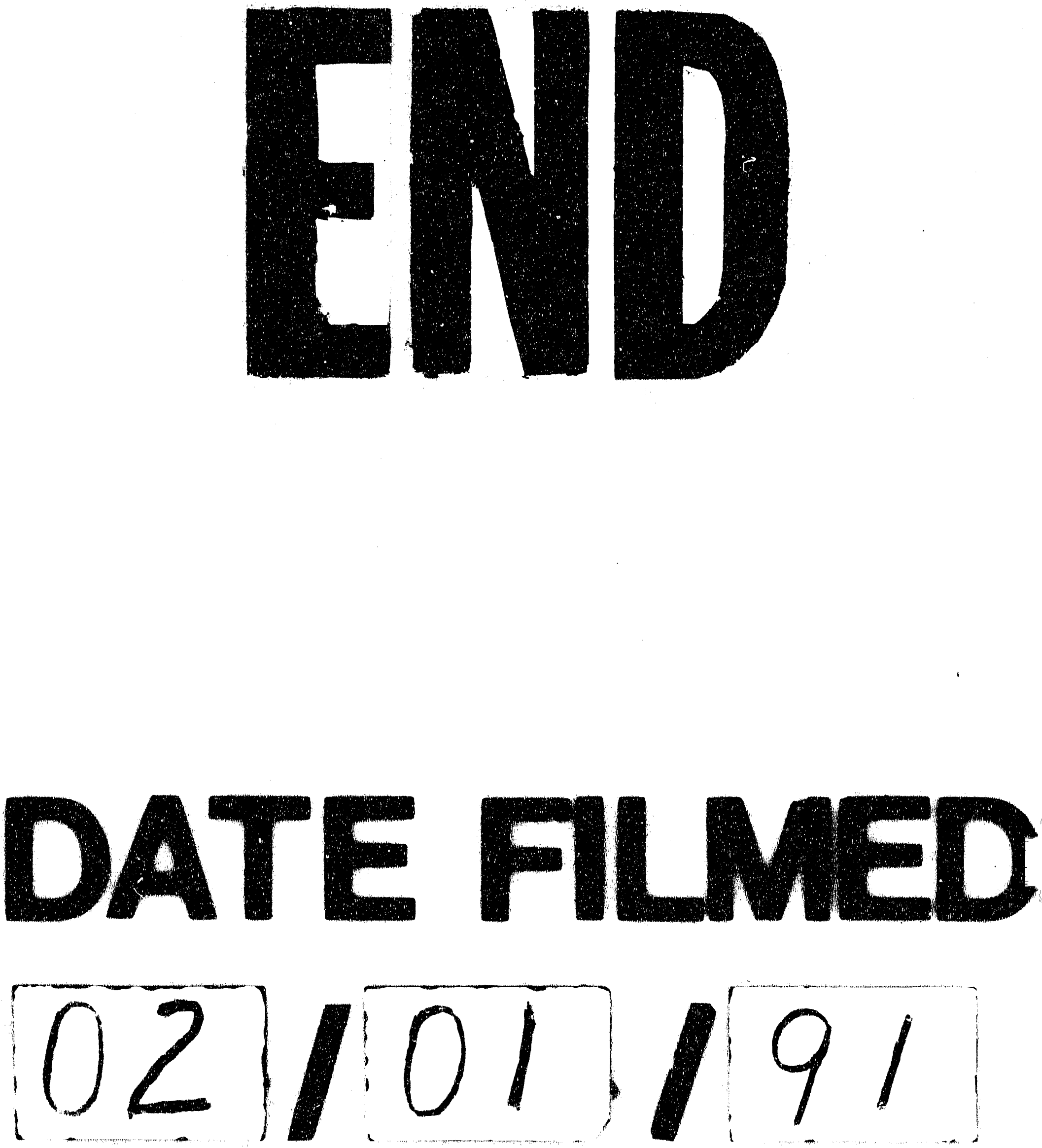
言 